Accepted Author Preprint, cite as: Rees N, John N, Vaughan N, Dorrington K, Day T, (2020) ParaVR: A Virtual Reality Training Simulator for Paramedic Skills maintenance, JPAR Journal of Paramedic Practice, November 2020 issue.

\title{
ParaVR: A Virtual Reality Training Simulator \\ for Paramedic Skills maintenance
}

\begin{abstract}
Background,

Virtual Reality (VR) technology is emerging as a powerful educational tool which is used in medical training and has potential benefits for paramedic practice education.
\end{abstract}

\section{Aim}

The aim of this paper is to report development of ParaVR, which utilises VR to address skills maintenance for paramedics.

\section{Methods}

University of Chester VR developers and the Welsh Ambulance Services NHS Trust (WAST) developed ParaVR in four stages: 1. Identifying requirements and specifications 2. Alpha version development, 3. Beta version development 4. Management: Development of software, further funding and commercialisation.

\section{Results}

Needle Cricothyrotomy and Needle Thoracostomy emerged as candidates for the prototype ParaVR. The Oculus Rift head mounted display (HMD) combined with Novint Falcon haptic device was used, and a virtual environment crafted using 3D modelling software, ported (a computing term meaning transfer (software) from one system or machine to another) onto Oculus Go and Google cardboard VR platform. 
Accepted Author Preprint, cite as: Rees N, John N, Vaughan N, Dorrington K, Day T, (2020) ParaVR: A Virtual Reality Training Simulator for Paramedic Skills maintenance, JPAR Journal of Paramedic Practice, November 2020 issue.

\section{Conclusion}

VR is an emerging educational tool with the potential to enhance paramedic skills development and maintenance. The ParaVR program is the first step in our development, testing, and scaling up of this technology.

Keywords: Paramedic, Virtual Reality, Simulation, Ambulance, Emergency

key points:

- VR technology is emerging as a powerful educational tool,

- $\quad$ VR is used in medical training.

- Needle Cricothyrotomy and Needle Thoracostomy emerged as candidates for our VR prototype.

- Oculus Rift head mounted display (HMD) combined with Novint Falcon haptic device was used in a virtual environment crafted using 3D modelling software. This was ported onto an Oculus Go and Google cardboard VR platform.

- Unstructured initial feedback received from paramedics was positive. However future robust generalisable research is required prior to adoption of VR in paramedic practice and education.

- The ParaVR program reported in this article is the first step in our development, testing and scaling up this technology for paramedic practice and education. 
Accepted Author Preprint, cite as: Rees N, John N, Vaughan N, Dorrington K, Day T, (2020) ParaVR: A Virtual Reality Training Simulator for Paramedic Skills maintenance, JPAR Journal of Paramedic Practice, November 2020 issue.

\section{Introduction}

The turn of the 21 st century marked a significant transition in the UK and international paramedic education from vocational training to higher education development (Cooper 2005). Paramedics now have developed their knowledge and clinical expertise across a wide range of specialities, including primary, urgent, unscheduled, emergency and critical care (CoP 2018). Whist this transition is based on a balanced approach to the integration of theory and practice to ensure competency (CoP 2017), simulation has long been a valuable method in paramedic education, involving techniques that imitate prehospital patient situations, and facilitating learning and development of psychomotor skills to demonstrate procedures, decision-making, and critical thinking (Jeffries 2005, Birt et al 2017a, Williams et al 2016 ).

Development, refinement, and mastery of clinical skills is often gained through initial teaching, learning and repeated clinical experience. However, retaining such skills is challenging, especially when they are infrequently used and with clinicians working in rural areas (Glazebrook \& Harrison 2006, Campbell et al, 2015, Coleman et al 2019). Campbell et al (2015) identified low levels of confidence with paramedics in skills they use rarely or infrequently, and believe they need to rehearse these skills by a variety of means, including through simulation at least yearly. VR technology is emerging as a powerful method across many areas of medical training and may be beneficial for paramedic education (Clare et al, 2017, Birt et al, 2017a,b, Theriault, 2017). However, due to the situated nature of care provided by paramedics, who often operate as a scattered workforce, with limited opportunities to practise skills, ParaVR focusses on maintenance of rarely performed skills, following initial training.

This article reports the development of ParaVR - Virtual Reality (VR) training for Paramedics, which is a collaboration between the Welsh Ambulance Services NHS Trust 
Accepted Author Preprint, cite as: Rees N, John N, Vaughan N, Dorrington K, Day T, (2020) ParaVR: A Virtual Reality Training Simulator for Paramedic Skills maintenance, JPAR Journal of Paramedic Practice, November 2020 issue.

(WAST) and the University of Chester Department of Computer Science. The ParaVR project uses VR to address maintenance of skills for rarely performed procedures by paramedics. This includes VR training for Needle Cricothyrotomy (NCCT) and Needle Thoracostomy (NT) decompression.

\section{Background}

Skills development and maintenance for many paramedic emergency procedures is challenging due to the limited opportunities to learn and practise. Such procedures may be required in rare and life-threatening situations, where they need to be delivered promptly and under stressful conditions. Paramedic practice and emergency care has therefore relied on simulation, which provides a unique opportunity for learners to practise clinical skills in a low-stakes setting before performing on real patients. Simulation is defined as:

"the imitation of tasks, relations, phenomena, equipments, behaviours and certain cognitive activities, which are present in reality" (Özkalpa \& Saygıli 2015)

Simulation is a technique which makes it possible to experience a real situation beforehand with the help of a guide (Gaba 2007). A wide range of models have long been used in paramedic practice and emergency care simulation, including animals, plastic models, modified commercial mannequins, paid or unpaid volunteers, patients recently pronounced dead, and cadavers (Nelson 1990, Bengiamin et al, 2019, Mc Ferguson et al, 2017). The uses of such models range from the introduction of Resusci Anne in the 1950's for Basic Life Support Training (Laerdal, 2019, Jones et al 2015, Simons 1986) to modern applications in areas such as resuscitative thoracostomy (Ferguson et al, 2017, Mc Ferguson et al, 2017) and extracorporeal cardio pulmonary resuscitation (ECPR) (Whitmore et al, 2019). Despite the many potential benefits of such models, practical and ethical concerns emerge, which are 
Accepted Author Preprint, cite as: Rees N, John N, Vaughan N, Dorrington K, Day T, (2020) ParaVR: A Virtual Reality Training Simulator for Paramedic Skills maintenance, JPAR Journal of Paramedic Practice, November 2020 issue.

discussed later in this article, along with the emerging potential of VR as an important simulation tool which may offer benefits for paramedic skills maintenance.

\section{VR in Clinical Simulation?}

A wide range of definitions exist for VR, and it has been suggested there is lack of standardization or coherence (Kardong-Edgren, et al 2019). When used in a nursing context,

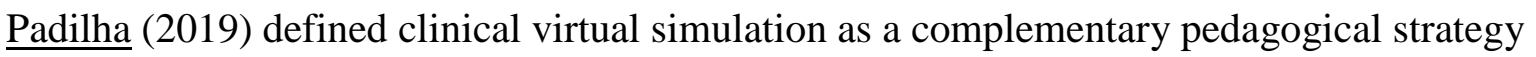
that provides the opportunity to improve clinical reasoning skills in students through exposure to many clinical scenarios. Cant et al, (2019) however, suggests a 3-step conceptual definition for VR, including level of fidelity, immersion, and patient depiction. KardongEdgren et al (2019) concurs with this definition and suggests it should be further delineated to include levels of immersion, which consider characteristics of presence, further advocating the adoption of standardized classification of VR levels, described as VR: low, VR:medium, VR: high. Based on this criteria, ParaVR was determined to be or VR: high, as it is immersive and includes features such as accommodating more than two sensory modalities (i.e. auditory, visual, motor/ proprioceptive); stimuli are spatially oriented, use of a headmounted device and the visual experience are altered to closely match proprioceptive feedback.

\section{Potential benefits of VR}

Health-care workers have long embraced and benefited from VR technology in a variety of areas, including surgical training where it has been shown to improve technical performance of surgical procedures (e.g. Seymour et al, 2002, Azarnoush et al, 2017, Davis et al, 2016, Nagendran et al, 2013). Members of the ParaVR team have extensive experience in developing such evidence-based VR applications in a variety of contexts, from minimally invasive medical procedures (John et al, 2015, Vaughan et al, 2016) to applying training for 
Accepted Author Preprint, cite as: Rees N, John N, Vaughan N, Dorrington K, Day T, (2020) ParaVR: A Virtual Reality Training Simulator for Paramedic Skills maintenance, JPAR Journal of Paramedic Practice, November 2020 issue.

powered wheelchair users (John et al, 2017). In the paramedic setting, the potential benefits of VR may include the following:

- Its ability to imitate all existing possibilities and provide a rich environment, where participants can respond realistically. Simulation should contain different paths that the participant can follow in case of a change in the problem or situation, and it should be able to act in accordance with the actions of the participant. The more of these features the system contains, the better the participants can transfer what they learned during the simulation to real life (Özkalp \& Sayg1l, 2015).

- VR can provide the ability for full immersion of the paramedic into a crafted, virtual world, which can be composed of a virtual patient, a model of the pathology, and all the surgical instruments needed. It can also be tailored to include an endless range of situations and options, thus reflecting the reality of experiences (Maran et al, 2003). The goal is for full immersion to replace the users' real-world surroundings convincingly enough so that they can suspend disbelief and fully engage with the created environment.

- VR can also provide feedback to students during training, making it possible for the student to learn from mistakes and gain experience without harming patients (Burgess, 2007).

- Even low-technology simulation tools are expensive and patient simulators often lack fidelity in terms of tactile feedback and appearance for optimal mastery of emergency procedures (Wang et al, 2007, Pettineo et al, 2009, Aggarwal et al, 2007). Basic mannequins often do not reflect patient variation such as age, weight, size, physiology etc and have limited real world accuracy (Perkins, 2007), they wear out. For instance, the cricothyroid membrane is puncturable and needs replacing frequently (John, 2007). They are also costly and only accessible at a few locations. 
Accepted Author Preprint, cite as: Rees N, John N, Vaughan N, Dorrington K, Day T, (2020) ParaVR: A Virtual Reality Training Simulator for Paramedic Skills maintenance, JPAR Journal of Paramedic Practice, November 2020 issue.

- The potential availability of VR at locations such as ambulance stations or Emergency Departments (ED’s) may increase access and opportunities for skills maintenance. This benefit has been found in emergency care due to the on-demand, user-driven method of learning in emergency care, rather than relying on the preparation, personnel and scheduling necessary for hands-on simulation sessions (Chang and Weiner, 2016).

- Learners can practise the simulation in their own time away from the clinical or classroom environment. This has significant potential in reducing time commitments for facilitators, reducing costs for universities/organisations, and reducing the number of resources required for simulation training (Ferguson et al, 2015; Chang and Weiner, 2016).

We therefore hypothesise that using immersive VR technologies with paramedics will add value to the paramedic training experience and enable support of skills retention.

\section{Reflexivity of authorship team:}

Our team includes a range of middle and senior level computer science academics whom have published widely in this area. The lead author (NR) is an Advanced Paramedic Practitioner and Ambulance Service/NHS research leader with thirty years' experience in prehospital care. He is an honorary lecturer at several Universities. This is important in terms of transparency, as the paper reports early phase development which requires further evaluation through research.

\section{Methods}

Following review against the Health Research Authority (HRA, 2019) guidance the project was not classed as research at this stage, but rather early phase innovation and prototype development. However WAST Research and Development forum maintain oversight of ParaVR, and future research will be required in order to produce generalisable findings. 
Accepted Author Preprint, cite as: Rees N, John N, Vaughan N, Dorrington K, Day T, (2020) ParaVR: A Virtual Reality Training Simulator for Paramedic Skills maintenance, JPAR Journal of Paramedic Practice, November 2020 issue.

A team of VR developers, researchers, paramedics and trainers from the University of Chester (UC) and the Welsh Ambulance Services NHS Trust (WAST) developed ParaVR in the following stages:

\section{Task 1: Requirements Specification}

UC and WAST worked collaboratively to specify the requirements for VR from the perspective of paramedic skills maintenance. These were documented and used to drive the development of the prototype system. This involved a literature review and gathering views from paramedics and training staff on the potential utility of VR for paramedic skills maintenance.

\section{Task 2: Development of alpha version of the VR}

At the outset of this project several affordable VR headsets were commercially available, including the Oculus Rift and Oculus Go. The Rift is a powerful device but has to be tethered by cables to an application computer. The Go is a tether-less device and is similar in performance to headsets that use a smartphone (such as the Google Cardboard). The Go does not need a separate smartphone, however, as this is integrated into the device. It was expected that a new device - the Oculus Quest - would be available during prototype development. The Quest is also tether-less but provides the same level of interaction as the Rift and would be a good choice for ParaVR. This demonstrates the fast-moving nature of VR technology development. An initial implementation prototype was produced for the two skills of NCCT and NT decompression. This was demonstrated to key stakeholders including 79 paramedics, training managers and student paramedics, and unstructured feedback was gathered.

\section{Task 3: Development of beta version of ParaVR}


Accepted Author Preprint, cite as: Rees N, John N, Vaughan N, Dorrington K, Day T, (2020) ParaVR: A Virtual Reality Training Simulator for Paramedic Skills maintenance, JPAR Journal of Paramedic Practice, November 2020 issue.

The beta version of ParaVR was further developed addressing the unstructured feedback obtained in Task 2, and porting the application to the Oculus Quest.

\section{Task 4: Management}

Along with the software development we also started investigating options for further research, funding, and the eventual commercialisation of ParaVR. It was decided that the prototype developed during this project would be utilised for leverage of further grant funding for the progression of the ParaVR program.

\section{Results}

\section{Task 1: Requirements Specification}

The two skills of NCCT and NT decompression were identified as candidates for the prototype ParaVR following the literature review and gathering of views from paramedics and training staff. This was due to these being infrequently performed life-saving paramedic skills and the potential utility of VR for skills maintenance.

\section{Literature for existing Paramedic VR training}

Hubble and Richards (2006) have previously demonstrated that paramedics can be trained at a distance, and Cone et al, (2011) indicated the efficacy of Virtual Reality as a platform for such distant paramedic education. Previous VR training simulators for paramedics have been developed (Conradi et al, 2009, Clare et al, 2017, Birt et al, 2017 a,b, Theriault, 2017), and participants in studies have reported that virtual patients delivered through a virtual world platform can provide a more authentic learner environment than classroom-based scenarios (Conradi et al, 2009). Mayrose et al, (2003, 2007) also previously developed a human airway 
Accepted Author Preprint, cite as: Rees N, John N, Vaughan N, Dorrington K, Day T, (2020) ParaVR: A Virtual Reality Training Simulator for Paramedic Skills maintenance, JPAR Journal of Paramedic Practice, November 2020 issue.

simulation model designed for tracheal intubation and again found it to be a useful educational tool.

So far, we have found no reports in the literature of a VR simulator to have incorporated paramedic NCCT or NT decompression. Some VR paramedic training simulators have been based in virtual environments such as train platforms or by the roadside (Conradi et al, 2009) or in a bus crash (Cone et al, 2011). The MESH360 project also presented a work-in-progress VR for paramedic life-and-death pressure situations or critical care (Cochrane et al, 2016).

\section{Needle Cricothyrotomy (NCCT)}

Needle Cricothyrotomy (NCCT) is a critical surgical intervention which may be life-saving during difficult airway management and a 'cannot ventilate, cannot intubate' situation to gain control of the airway that cannot otherwise be accessed in an emergency (Davies, 1999, Wong et al, 2003). If performed correctly, it is a quick and essential life-saving procedure. Catheter-over-needle cricothyrotomy seems to be a fast and easy procedure to perform (Vadodaria et al, 2004). However, most clinicians trained to conduct this procedure have only very limited experience with this technique, as only $1 \%$ of all critical airways require cricothyrotomy (Demirel et al, 2016), thus it is rarely used and if used, it is nearly always in a crisis situation, thus limiting trainee experience.

We found some basic existing simulation devices for general resuscitation which can support needle cricothyroidotomy among other procedures (Fig. 1). These are simple part-task or procedural trainers which are most commonly used to develop a basic psychomotor skill. No measurements were presented to demonstrate how realistic the feeling is compared to a real procedure on a live human.

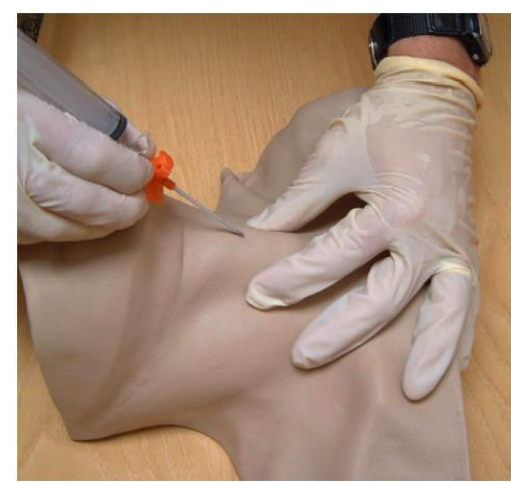

Fig 1. Part-task resuscitation trainer for NCCT (Perkins, 2007)

It was recognised that in such a NCCT procedure, haptic feedback of accuracy would be an 
Accepted Author Preprint, cite as: Rees N, John N, Vaughan N, Dorrington K, Day T, (2020) ParaVR: A Virtual Reality Training Simulator for Paramedic Skills maintenance, JPAR Journal of Paramedic Practice, November 2020 issue.

important feature. Porcine and human cadavers, although more realistic, also have their limitations, as the cricothyroid membrane becomes damaged with only one use.

\section{Needle Thoracostomy (NT) decompression}

NT decompression can be a life-saving procedure in trauma patients suffering from a tension pneumothorax, and is recommended by prehospital trauma guidelines (Kaserer et al, 2016, Leech et al, 2017, JRCALC, 2019). The technique for NT decompression requires knowledge of anatomic landmarks and a degree of surgical dexterity. The first choice of site is the 2 nd intercostal space (below the 2nd rib) in the mid-clavicular line, (MCL) (ICS2- MCL) and in the UK a standard 14G $4.5 \mathrm{~cm}$ long cannula is commonly used (Leech et al 2017). NT decompression is however again an infrequently performed skill by paramedics, indeed Kaserer et al, (2016) found that prehospital NT decompression was only performed in $1.1 \%$ of cases in a 6-year period involving 2261 severely injured patients. There are also possible complications which can result in failure of prehospital NT decompression to consider which can be modelled in the simulator. One of these failure reasons is the insufficient length of standard needles and catheters for the 2nd ICS to reach the intrapleural space; this is dependent on the morphology of the patient. When the needle/catheter reaches the pleural space pressure is applied allowing some release of gas and fluid but the catheter retracts into the intercostal muscle once the needle is withdrawn. Needles are also prone to kinking, or obstruction by blood or tissue. Some authors recommend the 5th ICS mid-axillar line for prehospital NT decompression due to the smaller chest wall thickness in this area to overcome these complications (Schroeder et al, 2013, Inaba et al, 2011). However, many UK ambulance services including WAST now use the Russell PneumoFix ${ }^{\circledR}$ (Prometheus Medical, 2019) which is designed and indicated specifically for this purpose $11 \mathrm{~cm}$ long 12 Gauge catheter - long enough to reach the pleural cavity of the vast majority of patients. The material chosen in Russell PneumoFix ${ }^{\circledR}$ also minimises the risk of kinking of the catheter. As 
Accepted Author Preprint, cite as: Rees N, John N, Vaughan N, Dorrington K, Day T, (2020) ParaVR: A Virtual Reality Training Simulator for Paramedic Skills maintenance, JPAR Journal of Paramedic Practice, November 2020 issue.

a general concept, obesity is increasingly prevalent in the population and the depth to the pleural space is likely to increase on a population level. This is related to previous research on VR modelling of various BMI patients which affects the depth of needle insertion (Vaughan et al, 2014).

\section{Task 2: Development of alpha version of the VR}

\section{A. Oculus Rift and Hands Interaction}

In our working prototype VR training simulator, the Oculus Rift head mounted display (HMD) was used. The Rift comes with wireless hand controllers (Fig. 2) which can be used by the learner or trainer to interact with the virtual model, pick up needles and insert the needle into the virtual patient. Although the learner is holding the controllers, only a model of a human hand is rendered in the virtual

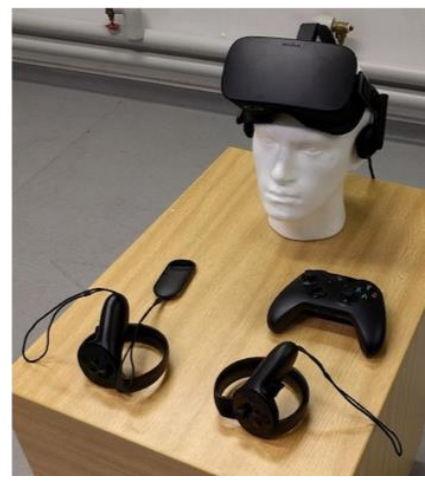

Figure 2. The Oculus Rift HMD with its interaction devices including wireless hand controllers

world. The controllers are ergonomically designed to allow the learner to point, grab, pick up, and interact with virtual objects in an intuitive fashion - and after a few minutes of use the learner forgets that they are interacting with a controller and it feels like they are using their own hands directly. When the needle is inserted, the needle stays in place after the hand controllers let go of the needle.

For haptic feedback, the VR simulator has also been combined with a Novint Falcon haptic device (Fig. 3). This provides three degrees of freedom (DOF) force feedback. As the learner moves the hand-held controller on the end of the interface, then the virtual needle follows its movement in the virtual world. This provides more fidelity for needle insertion as you can feel the sensation

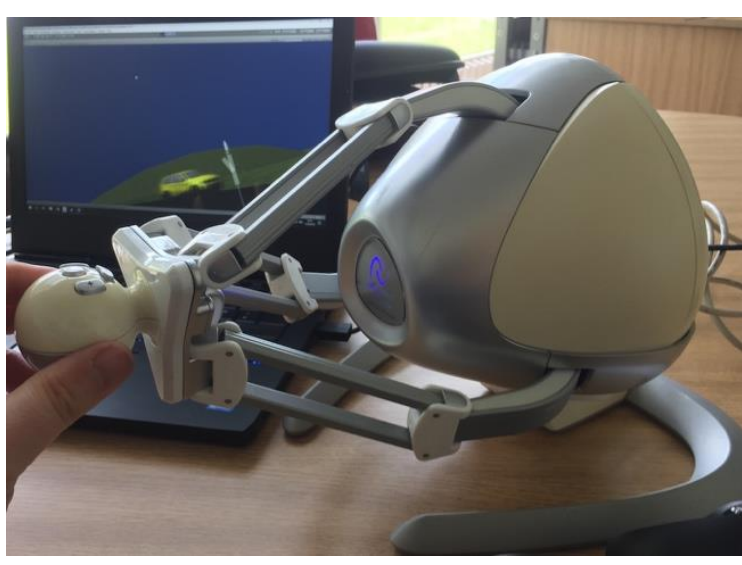

Figure 3: The Novint Falcon being used to control a virtual needle in ParavR 
of the needle puncture and an appropriate response as it penetrates different tissue types. The

Falcon has not been designed for use in immersive VR, however, and so integrating it seamlessly into the simulator is a challenge. It also adds to the cost of creating the simulator and can only be used if a separate computer is running ParaVR. It remains as an option that can be deployed when needed. The default mode is to use just the tracked hand controllers that come with the VR headset, as in Fig. 4.

\section{B. Virtual environment 3D software development}

The virtual environment for the paramedic simulator was designed to reflect the spontaneous nature of emergencies which could occur in unpredictable locations (Fig. 4). The environment was developed using 3D modelling software.

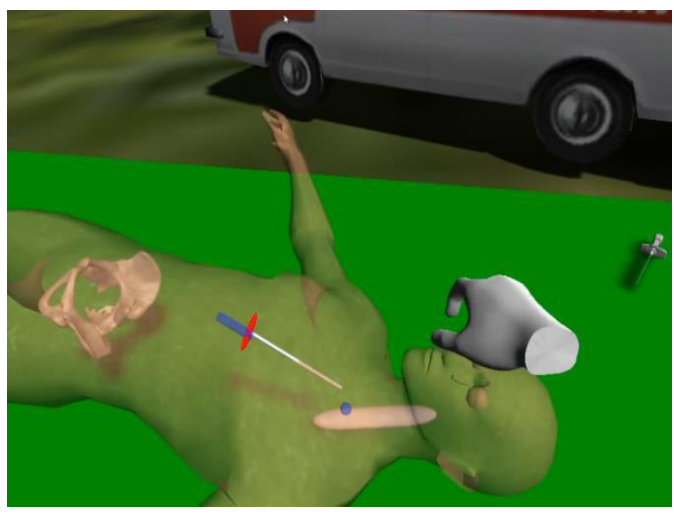
Additionally, the virtual training simulator was ported onto the Oculus Go and Google cardboard VR platform (Fig. 5)

Fig 4. VR model of ParaVR with Omni and Oculus Hand Controller.

to demonstrate the feasibility of paramedic training using a smartphone, without the need for a dedicated computer. However, these platforms do not support the use of a haptics device or dual hand controllers, and so the virtual model of the needle can only be picked up using a vision based interface which has less

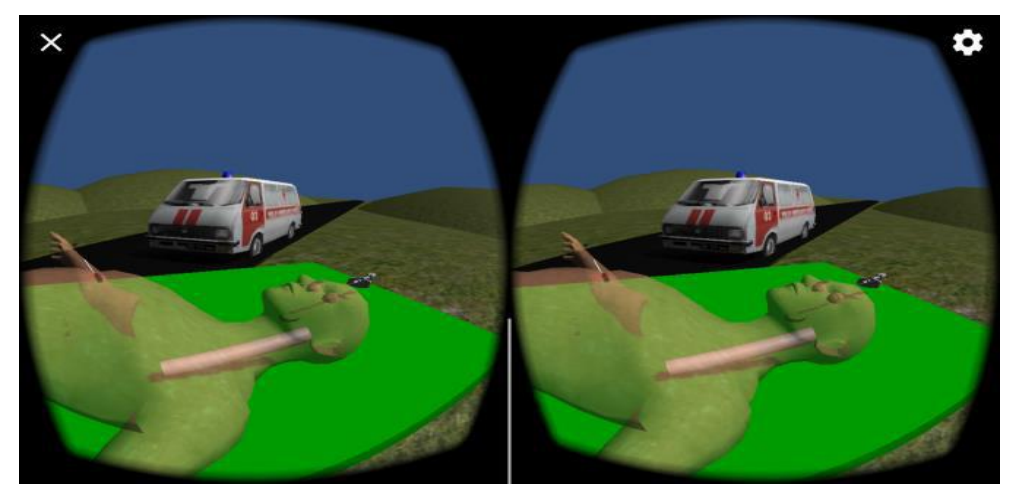
fidelity. 
Accepted Author Preprint, cite as: Rees N, John N, Vaughan N, Dorrington K, Day T, (2020) ParaVR: A Virtual Reality Training Simulator for Paramedic Skills maintenance, JPAR Journal of Paramedic Practice, November 2020 issue.

\section{A. Feedback from Paramedics}

Unstructured feedback has been provided by input from various paramedics and organization including WAST and Paramedic educators. ParaVR was demonstrated to 79 paramedics, and we continue to work closely with our supporting team members in the NHS and key stakeholders to demonstrate our developed system and gather feedback. We recognise that despite these activities and the potential benefits of VR, numerous complexities exist relating to the user interface, interoperability, and human factors (Kumar et al, 2011). Future research is therefore required to gather critical evidence and evaluation. This will further explore these factors along with the commercial potential, applicability and potential of the developed technology in the NHS.

\section{Discussion}

Simulation VR technology is increasingly used for training medical professionals and is anticipated to become more relevant in the setting of restricted clinical training hours, with a heightened focus on patient safety (McGrath et al, 2018). Paramedics often work as a scattered and mobile workforce, and skills maintenance may be a challenge. The UK College of Paramedics (CoP, 2018) encourages the use of simulated practice at all levels, and in its recently released Consensus Statement: A framework for safe and effective intubation by paramedics (CoP, 2018), they also contend that the use of simulation enables the development of competence not only in the technical, but also the non-technical skills related to undertaking intubation and associated decision-making, particularly in the prehospital setting.

Simulation must possess the property of fidelity, which can be defined as consistency with real life, or in other words, authenticity (Özkalpa \& Saygili, 2015). ParaVR presents an opportunity to imitate many existing possibilities and provide a rich environment, where participants can respond realistically. ParaVR can also be tailored to reflect anatomical 
Accepted Author Preprint, cite as: Rees N, John N, Vaughan N, Dorrington K, Day T, (2020) ParaVR: A Virtual Reality Training Simulator for Paramedic Skills maintenance, JPAR Journal of Paramedic Practice, November 2020 issue.

variations and introduce different paths that the paramedic can follow in responses to changes in the scenario. Features such as these, benefit the participants in simulation learning as they can transfer what they have learned during the simulation to the real life (Özkalp \& Saygıl, 2015).

We have argued that ParaVR has many potential advantages over traditional paramedic simulation learning, which includes the ability for full immersion of the paramedic into a crafted environment and the ability to experience stress situations without introducing risks to patients. Mannequins, animal models, and even human cadavers may be more realistic, however, they may also be impractical, as the membranes become damaged following use, they are expensive and may not be easily accessible.

\section{Limitations}

We opted for the techniques of NCCT and NT decompression for the initial development of ParaVR, as these are infrequently performed life-saving paramedic skills. However, more advanced skills such as tracheotomy, lateral thoracostomy, or tube thoracostomy may be more effective than the skills applied in ParaVR, but these are only performed by physicians in many emergency medical systems. Common alternative locations also exist for NT decompression, which includes the fourth (ICS4) and fifth (ICS5) intercostal spaces at both the anterior axillary line (AAL) (ICS4/5-AAL), and the midaxillary line (MAL) (ICS4/5MAL). Indeed, evidence from observational studies suggests that the 4th/5th ICS-AAL has the lowest predicted failure rate of NT decompression in multiple populations (Laan et al, 2016). Future changes in paramedic practice education may therefore involve alternative sites and more advanced techniques as skills and evidence evolves, which may make redundant some of the developments in ParaVR. However, this situation may also benefit from the flexibility of VR, as large scale inexpensive tailored software upgrades may be introduced in 
Accepted Author Preprint, cite as: Rees N, John N, Vaughan N, Dorrington K, Day T, (2020) ParaVR: A Virtual Reality Training Simulator for Paramedic Skills maintenance, JPAR Journal of Paramedic Practice, November 2020 issue.

the face of such emerging changes in practice in a way that expensive physical models cannot.

We also recognised the need for future research to gather critical evidence and evaluation prior to adoption.

\section{Next steps:}

The ParaVR team are currently collaborating with other UK Ambulance Trusts to attract further funding to determine whether ParaVR leads to more effective education through validation studies. The progression of this work therefore includes the need for a multicentre trial. Such future research and development of ParaVR may benefit from adopting an integrated systems design approach (Scerbo et al 2011).

\section{Conclusion}

VR simulation is an emerging educational tool and has important potential to enhance paramedic learning and skills maintenance. The ParaVR program of work reported in this paper is the first step in our development, testing and scaling up this technology for paramedic practice. The ParaVR program has followed four stages of development, which has included exploring the requirements specification, which identified NT decompression and NCCT as candidates for VR skills maintenance, we developed an alpha version of the VR, and following unstructured feedback, progressed to a more refined beta version of ParaVR. Future work will focus on demonstrating educational and cost-effective.

\section{Acknowledgment}

Funding was received to support the ParaVR project from Bevan Commission. The ParaVR concept was also the winner of the 2018 Health Gadget Hack organised by the Bevan Commission. The Royal Academy of Engineering provided a Research Fellowship funding award to Dr Neil Vaughan. 
Accepted Author Preprint, cite as: Rees N, John N, Vaughan N, Dorrington K, Day T, (2020) ParaVR: A Virtual Reality Training Simulator for Paramedic Skills maintenance, JPAR Journal of Paramedic Practice, November 2020 issue.

\section{References}

Aggarwal R, Ward J, Balasundaram I, Sains P, Athanasiou T, \& Darzi, A.. (2007) Proving the effectiveness of virtual reality simulation for training in laparoscopic surgery. Ann Surg 2007;246:771-9.

Azarnoush, H. Siar, S. Sawaya, R. Al Zhrani, G. Winkler-Schwartz, A. Alotaibi, F.E, Bajunaid K, Marwa I, Sabbagh AJ and Del Maestro, R.F.. The force pyramid: a spatial analysis of force application during virtual reality brain tumor resection. J Neurosurg 2017;127:171-81.

Bengiamin, D.I. toomasian, C. Smith, D.D. young, T.P. (2019) Emergency department thoracotomy: a cost-effective model for simulation training. The Journal of Emergency Medicine, vol. 57, no. 3, pp. 375-379, 2019

Birt, J., Moore, E., \& Cowling, M. (2017a.). Improving paramedic distance education through mobile mixed reality simulation. Australasian Journal of Educational Technology, 33(6). https://doi.org/10.14742/ajet.3596

Birt, J. Moore, E. Cowling, M.A. (2017) Piloting Mobile Mixed Reality Simulation in Paramedic Distance Education. Available from: http://www.segah.org/2017/docs/Papers/Session\%204\%20\%20Healthcare\%20Training\%20II/O-S04-3-07.pdf. [Accessed on 22/6/2019]

Campbell, D. Shepherd, I. McGrail, M. Kassell, L. Connolly, M. Williams, B. Nestel, D. (2015) Procedural skills practice and training needs of doctors, nurses, midwives and paramedics in rural Victoria Advances in Medical Education and Practice 2015:6 
Accepted Author Preprint, cite as: Rees N, John N, Vaughan N, Dorrington K, Day T, (2020) ParaVR: A Virtual Reality Training Simulator for Paramedic Skills maintenance, JPAR Journal of Paramedic Practice, November 2020 issue.

Cant, R. Cooper, S. Sussex, R. \& Bogossian, F. (2019) What's in a name? Clarifying the nomenclature of virtual simulation. Clinical Simulation in Nursing. 27, 26-30.

Chang, T.P. Weiner, D. (2016) Screen-based simulation and virtual reality for paediatric emergency medicine. Clin. Pediatr. Emerg. Med. 17, 224-230.

Chang, R.S. Hamilton, R.J. Carter, W.A. (1998) Declining rate of cricothyrotomy in trauma patients with an emergency medicine residency: implications for skills training. Acad Emerg Med 1998;5:247-51.

Cho, J. Kang, G.H. Kim, E.C, Oh Y. M, Choi H. J, Im T. H, Yang J. H, Cho Y. S, and Chung H. S. (2008) Comparison of manikin versus porcine models in cricothyrotomy procedure training. Emerg Med J 2008;25:732-4.

Clare, D, Rae, S. and Clarke, S. (2017) 'Can incorporating 360 degree video and virtual reality technology enhance the learning experience of paramedic students in distance education', Australasian Journal of Paramedicine, 14(1), p. 6.

Coleman, N. Barry, T. Tobin, H. Conroy, N. Bury, G· (2019) Paediatric airway management and concerns: a survey of advanced paramedics in Ireland. Ir J Med Sci. 2019 May;188(2):683-688. doi: 10.1007/s11845-018-1887-x.

Cone, D.C, Serra, J. Kurland, L. (2011) Comparison of the SALT and Smart triage systems using a virtual reality simulator with paramedic students. Eur J Emerg Med. Dec; 18 (6):31421. doi: 10.1097/MEJ.0b013e328345d6fd.

Cochrane, T, Cook, S, Aiello, S, Harrison, D, \& Aguayo, C. (2016). Designing virtual reality environments for paramedic education: MESH360. In Ascilite2016: 33rd International 
Accepted Author Preprint, cite as: Rees N, John N, Vaughan N, Dorrington K, Day T, (2020) ParaVR: A Virtual Reality Training Simulator for Paramedic Skills maintenance, JPAR Journal of Paramedic Practice, November 2020 issue.

Conference of Innovation, Practice and Research in the Use of Educational Technologies in Tertiary Education (pp. 125-135). Ascilite.

Conradi, E, Kavia, S, Burden, D, Rice, A, Woodham, L, Beaumont, C, ... \& Poulton, T. (2009). Virtual patients in a virtual world: Training paramedic students for practice. Medical Teacher, 31(8), 713-720.

Cooper, S. (2005) Contemporary UK paramedical training and education. How do we train? How should we educate? Emerg. Med. J. 22, 375e379

CoP (2017) Paramedic Curriculum Guidance 4th Edition - 2017. Available from: https://www.collegeofparamedics.co.uk/publications/professional-standards. [Accessed on 30/09/2018]

CoP (2018) Consensus Statement: A framework for safe and effective intubation by paramedics. Available from: https://www.collegeofparamedics.co.uk/publications. [Accessed on 30/09/2018]

Davis, D.P. Bramwell, K.J. Vilke, G.M. Cardall, T.Y. Yoshida, E. Rosen, P. Cricothyrotomy technique: standard versus the Rapid Four-Step Technique. Journal of Emergency Medicine 1999; 17: 17-21.DOI: 10.1016/S0736-4679(98)00118-8

Davis, M.C. Can, D.D. Pindrik, J. Rocque, B.G. Johnston, J.M. (2016) Virtual interactive presence in global surgical education: international collaboration through augmented reality. World Neurosurg. 2016;86:103-11.

Demirel, D. Butler, K.L. Halic, T, Sankaranarayanan, G, Spindler, D, Cao, C, Petrusa, E, Molina, M, Jones, D.B, De, S. and deMoya, M.A, (2016) A hierarchical task analysis of 
Accepted Author Preprint, cite as: Rees N, John N, Vaughan N, Dorrington K, Day T, (2020) ParaVR: A Virtual Reality Training Simulator for Paramedic Skills maintenance, JPAR Journal of Paramedic Practice, November 2020 issue.

cricothyroidotomy procedure for a virtual airway skills trainer simulator. The American Journal of Surgery, Vol 212, No 3, September 2016

Ferguson, C. Davidson, P.M. Scott, P.J. Jackson, D. Hickman, L.D. (2015) Augmented reality, virtual reality and gaming: an integral part of nursing. Contemp. Nurse 51, 1-4.

Fikkers, B.G. Van Vugt, S. Van Der Hoeven, J.D. Van Den Hoogen, F.J.A. Marres, H. A. M. Emergency cricothyrotomy: a randomised crossover trial comparing the wire-guided and catheter-over-needle techniques. Anaesthesia. Volume59, Issue10 October 2004

Gaba, D.M. (2007). The future of simulation in healthcare. Simulation in Healthcare, (2): $126-35$.

Garrett, B.M, Jackson, C, Wilson, B. (2015). Augmented reality m-learning to enhance nursing skills acquisition in the clinical skills laboratory. Interactive Technology and Smart Education, 12(4), 298-314.

Glazebrook R, Harrison S (2006) Obstacles to maintenance of advanced procedural skills for rural and remote medical practitioners in Australia. Rural Remote Health. 6(4):502.

HRA (2019) Health Research Authority: Is my study Research? Available from: http://www.hra-decisiontools.org.uk/research/ [Accessed on 10/07/2019]

Hubble, M.W. Richards, M.E. (2006) Paramedic Student Performance: Comparison of online with on-campus lecture delivery methods. Pre Hospital Disaster Medicine. DOI $10.1017 / \mathrm{s} 1049023 \times 00003800$

Inaba, K, et al. (2011) Optimal positioning for emergent needle thoracostomy: a cadaver based study. J Trauma 2011;71(5):1099-103 (discussion 1103). 
Accepted Author Preprint, cite as: Rees N, John N, Vaughan N, Dorrington K, Day T, (2020) ParaVR: A Virtual Reality Training Simulator for Paramedic Skills maintenance, JPAR Journal of Paramedic Practice, November 2020 issue.

Jeffries, P.R. (2005) A framework for designing, implementing, and evaluating simulations used as teaching strategies in nursing. Nurs Educ Perspect. 2005 Mar-Apr; 26(2):96-103.

John, B. Suri, I. Hillermann, C. Mendonca, C. (2007). Comparison of cricothyroidotomy on manikin vs. simulator: a randomised cross-over study. Anaesthesia, 62(10), 1029-1032.

John, N. W, Phillips, N. I, Cenydd, L. A, Coope, D, Carleton-Bland, N, Kamaly-Asl, I, \& Gray, W. P. (2015). A tablet-based virtual environment for neurosurgery training. Presence: Teleoperators and Virtual Environments, 24(2), 155-162.

John, N. W, Pop, S. R, Day, T. W, Ritsos, P. D, \& Headleand, C. J. (2017). The implementation and validation of a virtual environment for training powered wheelchair manoeuvres. IEEE transactions on visualization and computer graphics, 24(5), 1867-1878.

Jones F, Passos-Neto CE, Braguiroli OFM. (2015) Simulation in Medical Education: Brief history and methodology. PPCR 2015, Jul-Aug;1(2):56-63

JRCALC (2019) The Joint Royal Colleges Ambulance Liaison Committee 2019 Clinical Guidelines. Class Publishing; ISBN-13: 9781859596555

Kardong-Edgren, S. Farra, S. L. Alinier, G. Young, G.H (2019) A Call to Unify Definitions of Virtual Reality. Clinical Simulation In Nursing, Volume 31, 28 - 34

Kaserer, A. Hans-Peter, S. Donat, S. Spahna, R. Neuhaus, V. (2016) Failure rate of prehospital chest decompression after severe thoracic trauma. The American Journal of Emergency Medicine, Volume 35, Issue 3, 469 - 474

Kumar, S. Hedrick, M. Wiacek, C. Messner, J. I. (2011) Developing an experienced-based design review application for healthcare facilities using a 3d game engine. Journal of Information Technology in Construction, 16, 85-104. 
Accepted Author Preprint, cite as: Rees N, John N, Vaughan N, Dorrington K, Day T, (2020) ParaVR: A Virtual Reality Training Simulator for Paramedic Skills maintenance, JPAR Journal of Paramedic Practice, November 2020 issue.

Laan, D.V. Vu, T.D. Thiels, C.A. Pandian, T.K. Schiller, H.J. Murad, M.H. Aho, J.M. (2016)

Chest wall thickness and decompression failure: A systematic review and meta-analysis comparing anatomic locations in needle thoracostomy \& Injury. Int. J. Care Injured 47;797804

Laerdal (2019) Laerdal History. Available from:

https://www.laerdal.com/gb/docid/1117121/Laerdal-History [accessed on 12/11/2019]

Leech, C, Porter, K, Steyn, R, Laird, C, Virgo, I, Bowman, R, \& Cooper, D. (2017). The prehospital management of life-threatening chest injuries: A consensus statement from the Faculty of Pre-Hospital Care, Royal College of Surgeons of Edinburgh. Trauma, 19(1), 5462. https://doi.org/10.1177/1460408616664553

Liu, A. Bhasin, Y. \& Bowyer, M. (2005). A haptic-enabled simulator for cricothyroidotomy. Studies in Health Technology and Informatics. 111, 308-313.

Maran, NJ,. Glavin, RJ. (2003). Low-to High-Fidelity Simulation-A Continuum of medical education. Me Educ, 37: 22-28.

Mayrose, J. Kesavadas, T. Chugh, K. et al (2003) Utilization of virtual reality for endotracheal intubation training. Resuscitation. Oct;59(1):133-8.

Mayrose, J. Myers, J. (2007) Endotracheal Intubation: Application of Virtual Reality to Emergency Medical Services Education. Sim Healthcare 2:231-234, 2007

Mc Ferguson, I.M. Shareef, M.Z. Burns, B. Reid, C. (2016) A human cadaveric workshop: one solution to competence in the face of rarity. Emerg Med Australas 2016;28:752-4. 
Accepted Author Preprint, cite as: Rees N, John N, Vaughan N, Dorrington K, Day T, (2020) ParaVR: A Virtual Reality Training Simulator for Paramedic Skills maintenance, JPAR Journal of Paramedic Practice, November 2020 issue.

McGrath, J.L. Taekman, J.M.Parvati, D. Danforth, D.R. Mohan, D. Kman, N. Crichlow, A. Bond, W.F. (2018) Using Virtual Reality Simulation Environments to Assess Competence for Emergency Medicine Learners. Academic Emergency Medicine. 25:186-195.

Nagendran, M. Gurusamy, K.S. Aggarwal, R. Loizidou, M. \& Davidson, B.R. (2013). Virtual reality training for surgical trainees in laparoscopic surgery. The Cochrane Database of Systematic Reviews, 8, CD006575.

Nelson, M.S. (1990) Models for teaching emergency medicine skills. Ann Emerg Med. 1990 Mar;19 (3):333-5.

Özkalpa, B. Saygıli, U. (2015) The effectiveness of similitor usage in the paramedic education. Procedia - Social and Behavioral Sciences 174; 3150 - 3153

Padilha, J.M. Machado, P.P Ribeiro, A. Ramo, J. Costa, P. (2019) Clinical Virtual Simulation in Nursing Education: Randomized Controlled Trial. J Med Internet Res. vol. 21. iss. 3 | e11529.p. 1

Perkins, G. D. (2007). Simulation in resuscitation training. Resuscitation, 73(2), 202-211.

Pettineo, C.M. Vozenilek, J.A. Wang, E, et al. (2009) Simulated emergency department procedures with minimal monetary investment: cricothyrotomy simulator. Simul Health $2009 ; 4: 60-4$

Prometheus Medical (2019) The Russell PneumoFix. Available from: https://www.prometheusdeltatech.com/product/russell-pneumofix/ [accessed on 28/07/209] Scerbo, M.W. Murray, W.B. Alinier, G. Antonius, T. Caird, J. Stricker, E. Rice, J. Kyle, R (2011) A Path to Better Healthcare Simulation Systems: Leveraging the Integrated Systems Design Approach. Sim Healthcare; 6:S20-S23, 2011 
Accepted Author Preprint, cite as: Rees N, John N, Vaughan N, Dorrington K, Day T, (2020) ParaVR: A Virtual Reality Training Simulator for Paramedic Skills maintenance, JPAR Journal of Paramedic Practice, November 2020 issue.

Schroeder E, et al. (2013) Average chest wall thickness at two anatomic locations in trauma patients. Injury 2013;44(9):1183-5.

Seymour, N.E. Gallagher, A.G. Roman, S.A. O'Brien, M.K. Bansal, V.K.

Andersen, D.K. et al. (2002) Virtual reality training improves operating room performance: results of a randomized, double-blinded study. Annals of Surgery, 236 (4) (2002), pp. 458463 discussion 463-464

Simons, S.R. (1986) ABC of Resuscitation: Training Manakins. British Medical Journal. Volume : 292. 7 June 1986

Theriault, R. (2017) 'Virtual Reality in Paramedic Education', Canadian Paramedicine, 40(7), pp. 12-13.

Vadodaria, B.S. Gandhi, S.D. McIndoe, A.K. Comparison of four different emergency airway access equipment sets on a human patient simulator. Anaesthesia 2004; 59: 73-9.DOI: 10.1111/j.1365-2044.2004.03456.x

Vaughan, N, Dubey, V.N, Wee, M.Y. and Isaacs, R, 2014. Parametric model of human body shape and ligaments for patient-specific epidural simulation. Artificial intelligence in medicine, 62(2), pp.129-140.

Vaughan, N, Dubey, V. N, Wee, M. Y. K, and Isaacs, R. Mechanism for Adaptive Virtual Reality Feedback. ASME. J. Med. Devices. September 2016; 10(3): 030951

Wang, E.E. Vozenilek, J.A. Flaherty, J. et al. (2007) An innovative and inexpensive model for teaching cricothyrotomy. Simul Health. 2: 25-9

Whitmore, S.P. Gunnerson, K.J. Haft, J.W. Lynch, W.R. VanDyck, T. et al (2019) Simulation training enables emergency medicine providers to rapidly and safely initiate extracorporeal 
Accepted Author Preprint, cite as: Rees N, John N, Vaughan N, Dorrington K, Day T, (2020) ParaVR: A Virtual Reality Training Simulator for Paramedic Skills maintenance, JPAR Journal of Paramedic Practice, November 2020 issue.

cardio pulmonary resuscitation(ECPR) in a simulated cardiac arrests scenario. Resuscitation. May 2019 Vol. 138;p.68-73

Williams, B. Abel, C. Khasawneh, E. Ross, L. Levett-Jones, T. (2016) Simulation experiences of paramedic students: a cross-cultural examination. Advances in Medical Education and Practice 2016:7 181-186

Wong, D.T. Prabhu, A.J. Coloma, M. et al (2003) What is the minimum training required for successful cricothyroidotomy? A study in mannequins. Anesthesiology; 98:349-53. 